\title{
Distribution of affected muscles and degree of neurogenic lesion in patients with spina bifida
}

Ivana Petronic ${ }^{1,2}$, Dejan Nikolic', Dragana Cirovic ${ }^{1,2}$, Suzana Cvjeticanin ${ }^{2,3}$, Tatjana Knezevic', Mirjana Raicevic ${ }^{4}$, Radivoj Brdar ${ }^{2,4}$, Dragana Dzamic ${ }^{1}$, Nenad Janic ${ }^{2,4}$, Zoran Golubovic ${ }^{2,4}$

1Physical Medicine and Rehabilitation Department, University Children's Hospital, Belgrade, Serbia

2Faculty of Medicine, University of Belgrade, Serbia

3 Institute for Human Genetics, School of Medicine, Belgrade, Serbia

4Pediatric Surgery Department, University Children's Hospital, Belgrade, Serbia

Submitted: 25 October 2010

Accepted: 13 March 2011

Arch Med Sci 2011; 7, 6: 1049-1054

DOI: 10.5114/AOMS.2011.26619

Copyright $\odot 2011$ Termedia \& Banach
Corresponding author: Dejan Nikolic MD, PhD

University Children's Hospital Tirsova 10, 11000 Belgrade,

Serbia

Phone: +381638133345

E-mail: denikol27@yahoo.com

\begin{abstract}
Introduction: Patients with spina bifida in the lumbosacral region usually have various degrees of motor and sensory dysfunctions of the lower extremities and anal sphincter. The aim of our study was to evaluate the distribution and differences in frequencies of affected muscles, number of affected muscles and degree of neurogenic lesion between patients with spina bifida occulta (SBO) and spina bifida aperta (SBA).

Material and methods: In 100 patients with SB, 6 muscles in the lower limbs were separately analysed. Due to the number of affected muscles, we evaluated 5 groups of patients: with 1 affected muscle, 2 affected muscles, 3 affected muscles, 4 affected muscles and 5 affected muscles. Three degrees of neurogenic lesions were assessed: mild, moderate and severe.

Results: The tibialis anterior muscle was most frequently affected in SB patients. The outer anal sphincter was frequently affected in the group of SBA patients. Single muscle affection is frequent in the group of patients with $\mathrm{SBO}$, while in the group of patients with SBA, 4 muscles were significantly frequently affected. The great majority of patients (45.46\%) with affected outer anal sphincter (OAS) in the group of SBO were without affection of other muscles, while for the SBA group it was for every third patient. Mild neurogenic lesion was significantly frequent in SBO patients, while severe form was significantly frequent in SBA patients.

Conclusions: Patients with SBO usually present with mild to moderate clinical presentation, while multiple root involvement and severe degree of neurogenic lesion is associated more frequently with SBA.
\end{abstract}

Key words: spina bifida occulta, spina bifida aperta, muscles, electromyography, neurogenic lesion.

\section{Introduction}

Spina bifida (SB) or spinal dysraphism (SD) presents as failure of neural tube closure during neurulation [1]. It can be classified as spina bifida occulta (SBO) and spina bifida aperta (SBA) [2]. Patients with SB in the lumbosacral region usually have various degrees of motor and sensory dysfunctions of the legs and/or improper function of the anal and urethral sphincter [1]. There are authors who stress that motor development and muscle innervations are influenced by the level of neurological impairment [3]. 
Beside numerous studies that investigated the aetiology of SB $[4,5]$, there is a lack of data in the literature regarding evaluation of affected muscles and differences in their distribution between subentities of SB as well as evaluation of the degree of neurogenic lesion in SBO and SBA patients.

A sensitive method for evaluation of muscle function and therefore its affection in various conditions is electromyography (EMG). Stalberg reported a strong correlation between nerve and muscle disorders and changes in organization of muscle fibres [6]. Beside morphological techniques, these changes can be studied by EMG evaluation. Electromyographic recordings are dependent on the number and the size of muscle fibres and transmission of action potentials [6]. Also, EMG as a sensitive tool can assess accurately damage of peripheral motor pathways in patients with an affected spinal cord [7]. Therefore, EMG as a diagnostic technique is useful in detection of muscle affection presence and assessment of neurogenic lesion degree.

In order to gain more information regarding pathology of SB, the aim of our study was to assess distribution of affected muscles in patients with SB and to evaluate differences in frequencies of affected muscles, number of affected muscles and degree of neurogenic lesion between patients with SBO and SBA.

\section{Material and methods}

\section{Study group}

In our study we evaluated 100 patients with SB who were diagnosed and treated at University Children's Hospital (UCH) in Belgrade. All patients were clinically assessed and diagnosis of SB was confirmed by radiological and imaging tools (magnetic resonance imaging - MRI). Classification of SB into two entities, SBO and SBA, was done according to clinical and morphophysiological signs (increased density of hair and/or changes in skin pigmentation in lumbosacral region, gluteus line asymmetry, presence of fovea spinalis, lipomatosis, etc.) and radiological or imaging findings. The representative population within the study included non-surgically treated SBO and surgically treated SBO and SBA participants. The criteria for inclusion of patients with SBO were presence of morphophysiological signs and/or clinical pathology (weakness in lower limbs, foot deformities or sphincter dysfunctions) and confirmation with radiographic or imaging findings, while the criterion for inclusion of patients with SBA was clinical presentation of anomaly in the lumbosacral region confirmed with radiographic or imaging findings. Only operated SBA patients were included and evaluated. The criteria for exclusion were negative findings on radiographic and/or imaging tools for SBO, non- operated SBA patients and cervical or thoracic level of spinal dysraphism.

As the referral multidisciplinary centre for paediatric diseases, deformities and congenital anomalies in Serbia, those children were referred to $\mathrm{UCH}$ from maternity hospitals and other primary health care centres for initial screening, evaluation and treatment without dissemination and dropping out during the course of follow-up.

Individual patient characteristics were evaluated separately for SBO and SBA groups: age at EMG evaluation, gender, operated individuals, age at operation, presence of foot deformities, hydrocephalus, paresis, paralysis, incontinence and enuresis/encopresis.

For evaluation and diagnosis of affected muscles in the observed population we used needle electromyography to detect the potential presence of denervation potentials [8]. The responses were recorded and presented on the monitor. Six different muscles were analysed: outer anal sphincter (OAS), tibialis anterior (TA), extensor digiti brevi (EDB), gastrocnemius medialis (GM), rectus femoris (RF) and pollicis longus (PL). According to the number of affected muscles we evaluated 5 groups of patients: with 1 affected muscle (first group), 2 affected muscles (second group), 3 affected muscle (third group), 4 affected muscles (fourth group) and 5 affected muscles (fifth group).

The population with affected OAS was separately evaluated in order to establish its frequency. In the same population, we wanted to evaluate the correlation of the number of other affected muscles separately for SBO and SBA patients. Therefore, this population was classified into 5 groups: with only OAS affected, with 1 affected muscle beside OAS, and with 2, 3 and 4 affected muscles beside OAS.

Clinical and EMG findings were used to determine the type and degree of neurogenic lesion. Patients were placed into 2 groups regarding the type of neurogenic lesion: a group with paresis and a group with paralysis of the lower extremities. Participants with anal sphincter affection were placed into two groups: a group with incontinence and a group without encopresis. Presence of paresis, paralysis, incontinence and encopresis was analysed by EMG of evaluated muscles of lower extremities and OAS. Regarding the degree of neurogenic lesion, patients were classified into 3 groups: with mild neurogenic lesion, with moderate neurogenic lesion, and with severe neurogenic lesion.

To assess the degree of neurogenic lesion we applied neurophysiological examination that included EMG of certain muscles. To determine muscle activity by needle EMG we used a standardized procedure that included analysis after needle insertion 
and spontaneous activity, as well as voluntary motor-unit action potentials [9].

\section{Statistical analysis}

To present gender distribution, distribution of operated children, foot deformities, hydrocephalus, paresis, paralysis, incontinence and enuresis/encopresis, distribution of affected muscles, groups of muscles, and type and degree of neurogenic lesion in evaluated patients we used percentage values. The mean value (MV) with standard error of the mean (SEM) was used for the number of affected muscles overall and the number of affected muscles in the group of patients with affected OAS. To compare gender distribution, and type of neurogenic lesion distribution (paresis versus paralysis; incontinence versus enuresis/encopresis) within the same subgroups (SBO and SBA) we used $\chi^{2}$ test. To compare distribution of operated patients, foot deformities and hydrocephalus as well as distribution of affected muscles, groups of affected muscles and degree of neurogenic lesion between SBO and SBA patients we used $\chi^{2}$ test. For comparison of number of affected muscles between SBO and SBA patients we used Student's $t$ test.

\section{Results}

Of 100 patients with diagnosed spinal dysraphism, 50 had SBO and 50 had SBA. All patients were evaluated separately by the EMG diagnostic tool in order to establish affection of certain muscles.

In Table I, patients characteristics are presented separately for those with SBO and for those with SBA. It is shown that female gender was frequent but not significantly in both groups of patients $(p>0.05)$, while a significantly lower proportion of patients with SBO $(p<0.01)$ required surgery. Foot deformities and hydrocephalus were significantly frequent $(p<0.01)$ in the group of patients with SBA. Paresis and enuresis/encopresis were frequent in both groups of patients but significantly $(p<0.01)$ in the group of SBO patients.

Skin markers as morphophysiological changes were present in 36 children (72\%) with SBO.
Patients with SBO if indicated were surgically treated between 3 months of life and 12 years, while those with SBA were surgically treated within the first week of life. The age of SBO participants at EMG evaluation ranged between 3 months and 18 years, since in later stages of growth and development, particularly in puberty, SBO without previous clinical signs and symptoms could present with them. Participants with SBA were evaluated by EMG in the period from birth to 12 months of life.

Percentage distribution of evaluated muscles in groups of patients with SB and its entities (SBO and SBA) is separately assessed in Table II. We found that of all evaluated muscles, in $33 \%$ of patients the OAS was affected, indicating that OAS affection is expected in every third patient with spinal dysraphism. The tibialis anterior muscle was affected in almost $2 / 3$ of the patients with SB (68\%), more frequently in SBA patients but not significantly. Our

Table I. Presentation of patient characteristics of different subgroups

\begin{tabular}{|c|c|c|}
\hline Patient characteristics & $\begin{array}{c}\mathrm{SBO} \\
N=50, n(\%)\end{array}$ & $\begin{array}{c}\text { SBA } \\
N=50, n(\%)\end{array}$ \\
\hline \multicolumn{3}{|l|}{ Gender } \\
\hline Male & $21(42)$ & $24(48)$ \\
\hline Female & $29(58)$ & $26(52)$ \\
\hline Operated children & $6(12)$ & $50(100)^{*}$ \\
\hline Foot deformities & $27(54)$ & $50(100)^{*}$ \\
\hline Hydrocephalus & $0(0)$ & $36(72)^{*}$ \\
\hline $\begin{array}{l}\text { Patients with affected } \\
\text { muscles on lower } \\
\text { extremities }\end{array}$ & $\begin{array}{c}\mathrm{SBO} \\
N=45, n(\%)\end{array}$ & $\begin{array}{c}\text { SBA } \\
N=43, n(\%)\end{array}$ \\
\hline Paresis & $44(97.78)$ & $23(53.49)$ \\
\hline Paralysis & $1(2.22)^{*}$ & $20(46.51)$ \\
\hline $\begin{array}{l}\text { Patients with OAS } \\
\text { affection }\end{array}$ & $\begin{array}{c}\mathrm{SBO} \\
N=11, n(\%)\end{array}$ & $\begin{array}{c}\text { SBA } \\
N=22, n(\%)\end{array}$ \\
\hline Incontinence & $2(18.18)$ & $9(40.91)$ \\
\hline Enuresis/encopresis & $9(81.82)^{*}$ & $13(59.09)$ \\
\hline
\end{tabular}

OAS - outer anal sphincter, SBO - spina bifida occulta, SBA - spina bifida aperta, ${ }^{*} p<0.01$

Table II. Distribution of affected muscles in patients with spina bifida and its entities (SBO and SBA)

\begin{tabular}{|lcccc|}
\hline Muscles & SB & SBO & SBA & $N=50, n(\%)$ \\
\hline Outer anal sphincter & $33(33)$ & $11(22)$ & $22(44)$ & $\begin{array}{c}\chi^{2} \\
(S B O / S B A)\end{array}$ \\
\hline Tibialis anterior & $68(68)$ & $31(62)$ & $37(74)$ & $5.47^{*}$ \\
\hline Extensor digiti brevi & $55(55)$ & $25(50)$ & $30(60)$ & 1.65 \\
\hline Gastrocnemius medialis & $58(58)$ & $27(54)$ & $31(62)$ & 1.01 \\
\hline Rectus femoris & $13(13)$ & $4(8)$ & $9(18)$ & 0.68 \\
\hline Pollicis longus & $8(8)$ & $3(6)$ & $5(10)$ & 2.21 \\
\hline
\end{tabular}

SB - spina bifida, SBO - spina bifida occulta, SBA - spina bifida perta, ${ }^{*} p<0.05$ 
results indicated that OAS affection is significantly frequent in patients with SBA $\left(\chi^{2}=5.47 ; p<0.05\right)$.

Percentage distribution of number of affected muscles in groups of patients with SBO and SBA is separately assessed in Table III. There is a discrepancy between the first three groups and the last two regarding the number of patients and distribution of affected muscles. There was a higher proportion of patients with SBO in the groups with 1, 2 and 3 affected muscles, while a higher proportion of patients with SBA was observed in the groups with 4 and 5 affected muscles. In the group of patients with SBA, four muscles were significantly frequently affected $\left(\chi^{2}=6.06 ; p<0.05\right)$.

In Table IV, we present the percentage distribution of number of affected muscles in the group of OAS affected patients with SBO and SBA. Our results show that the great majority of patients $(45.46 \%)$ with affected OAS in the group of SBO were without affection of other muscles at the time of EMG diagnosis, while for the SBA group it was the case for every third patient. We found that three and four additional affected muscles beside OAS were more frequent in the group with SBA but without statistical significance.

We show in Table $V$ that the mean number of affected muscles is in the range between 2 and 3 muscles, with a statistically higher number of

Table III. Distribution of number of affected muscles according to the type of evaluated spinal dysraphism

\begin{tabular}{|lccc|}
\hline $\begin{array}{l}\text { Number of affected } \\
\text { muscles }\end{array}$ & $\begin{array}{c}\text { SBO } \\
N=50, n(\%)\end{array}$ & $\begin{array}{c}\text { SBA } \\
N=50, n(\%)\end{array}$ & $\chi^{2}$ \\
\hline One muscle & $16(32)$ & $11(22)$ & 1.27 \\
\hline Two muscles & $15(30)$ & $12(24)$ & 0.46 \\
\hline Three muscles & $16(32)$ & $13(26)$ & 0.44 \\
\hline Four muscles & $2(4)$ & $10(20)$ & $6.06^{*}$ \\
\hline Five muscles & $1(2)$ & $4(8)$ & 1.90 \\
\hline
\end{tabular}

SBO - spina bifida occulta, SBA - spina bifida perta, ${ }^{*} p<0.05$

Table IV. Distribution of number of affected muscles in group of OAS affected patients according to the type of evaluated spinal dysraphism

\begin{tabular}{|lccc|}
\hline OAS & $\begin{array}{c}\text { SBO } \\
N=11, n(\%)\end{array}$ & $\begin{array}{c}\text { SBA } \\
N=22, n(\%)\end{array}$ & $\chi^{2}$ \\
\hline Only OAS & $5(45.46)$ & $7(31.82)$ & 0.60 \\
\hline $\begin{array}{l}\text { OAS and one } \\
\text { muscle }\end{array}$ & $1(9.09)$ & $1(4.54)$ & 0.27 \\
\hline $\begin{array}{l}\text { OAS and two } \\
\text { muscles }\end{array}$ & $3(27.27)$ & $5(22.73)$ & 0.08 \\
\hline $\begin{array}{l}\text { OAS and three } \\
\text { muscles }\end{array}$ & $1(9.09)$ & $6(27.27)$ & 1.45 \\
\hline $\begin{array}{l}\text { OAS and four } \\
\text { muscles }\end{array}$ & $1(9.09)$ & $3(13.64)$ & 0.14 \\
\hline
\end{tabular}

$O A S$ - outer anal sphincter, SBO - spina bifida occulta, SBA - spina bifida aperta affected muscles $(t=2.37 ; p<0.05)$ for the group of patients with SBA. In the group of SBA patients with affected OAS, there is a higher number of other affected muscles but not significantly.

Regarding distribution of the degree of neurogenic lesion in patients with SBO and SBA (Table VI) it is shown that mild degree of neurogenic lesion is significantly more frequent in those with SBO $\left(\chi^{2}=12.15 ; p<0.01\right)$, while severe degree is observed to be significantly frequent in the group with SBA $\left(\chi^{2}=9.01 ; p<0.01\right)$.

\section{Discussion}

Instead of manual muscle testing (MMT), we have used EMG for muscle affection investigation due to several advantages: there is no age limitation, unlike MMT [3]; isolated muscle evaluation can be done with objective results and there is confident accuracy to determine neurological level.

We have pointed out that more severe clinical and neurological manifestations are related to SBA (frequent paralysis, foot deformities, hydrocephalus and incontinence). In the study of Dias, it was stressed that severity of pathological changes that are present in the group of SBA patients is justified by the fact that this entity is the most frequent congenital neurological malformation that is compatible with the living [10].

In the majority of patients with SBO (72\%) skin markers were present. These findings are consistent with previous reports [11]. Since a certain proportion of children with SBO could present without clinical manifestations (weakness, deformities and sphincter dysfunctions), it is of great importance to follow up regularly these children during growth

Table V. Mean value of number of affected muscles in patients with spinal dysraphism

\begin{tabular}{|lccc|}
\hline & $\begin{array}{c}\text { SBO } \\
(\mathrm{MV} \pm \mathrm{SEM})\end{array}$ & $\begin{array}{c}\text { SBA } \\
(\mathrm{MV} \pm \mathrm{SEM})\end{array}$ & $\begin{array}{c}\text { T test } \\
(t \text { value })\end{array}$ \\
\hline Affected muscles & $2.14 \pm 0.14$ & $2.68 \pm 0.18$ & $2.37^{*}$ \\
\hline $\begin{array}{l}\text { Affected muscles } \\
\text { in OAS affected } \\
\text { patients }\end{array}$ & $2.33 \pm 0.42$ & $2.73 \pm 0.23$ & 0.89 \\
\hline
\end{tabular}

OAS - outer anal sphincter, SBO - spina bifida occulta, SBA - spina bifida aperta, MV - mean value, SEM - standard error of mean, ${ }^{*} p<0.05$

Table VI. Distribution of degree of neurogenic lesion in patients with spina bifida

\begin{tabular}{|lccc|}
\hline $\begin{array}{l}\text { Degree of } \\
\text { neurogenic } \\
\text { lesion }\end{array}$ & $\begin{array}{l}\text { SBO } \\
50, n(\%)\end{array}$ & $\begin{array}{c}\text { SBA } \\
N=50, n(\%)\end{array}$ & $\chi^{2}$ \\
\hline Mild & $28(56)$ & $11(22)$ & $12.15^{\star}$ \\
\hline Moderate & $13(26)$ & $16(32)$ & 0.44 \\
\hline Severe & $9(18)$ & $23(46)$ & $9.01^{*}$ \\
\hline
\end{tabular}

$S B O$ - spina bifida occulta, $S B A-$ spina bifida perta, ${ }^{*} p<0.01$ 
and development and to establish timely diagnosis of tethered cord in order to prevent possible complications that could present later. Since MRI is a non-invasive and radiation-free diagnostic method, some authors suggest its use as firstchoice examination for the detection of SBO [11].

Significantly increased frequency of incontinence, especially in SBA patients, could be explained by abnormality of rectosigmoid compliance and rectoanal reflex as well as the loss of rectoanal sensibility [12].

In our study we have demonstrated that the most frequently affected muscle in both SBO and SBA groups of patients was TA, in almost two out of three patients with SBO and three quarters of patients with SBA. More than half of participants in both subentities of SB had affected GM and/or EDB muscles.

Regarding the number of affected muscles, we have found that one, two or three muscles were frequently affected in the group of patients with SBO. Opposite to these findings, four and five muscles were more frequent in patients with SBA, where four muscles had significantly higher affection. Such findings are consistent with the observations of Hong regarding multiple root affection in patients with myelomeningocele [13].

There are studies indicating an association between presence of spinal dysraphism and faecal incontinence or constipation $[14,15]$. Some aspects of these functional disorders are in direct correlation with status of OAS integrity and function. Even though we have found that OAS affection is frequent in children with SBA, isolated OAS affection is more often observed in patients with SBO, implying that bowel and defecatory dysfunctions are associated with increased incidence of SBO. Such claims are consistent with other researchers' observations [16]. Affection of other muscles in our survey for SBA patients with affected OAS is frequently seen, particularly 2 and 3 affected muscles, concerning almost every second patient (50\%) with such a state. Contrary to these findings, the most common number of affected muscles for SBO patients with affected OAS is 2, concerning nearly every fourth patient. This clearly stresses the fact that patients with SBA as a severe type of SB usually present with more complex clinical and functional conditions. Therefore, for complete assessment of SB children with affected OAS, integrity and functional status of other muscles should be evaluated, particularly in those with SBA.

Our investigation demonstrated that patients with SBA, almost two out of three, present with multiple root involvement, contrary to the group with SBO, where we found that every second patient has multiple root involvement. As previously stated regarding the number of affected muscles, these findings are also consistent with Hong's observations [13].

We have pointed out that more than half of patients with diagnosed SBO (56\%) present with mild degree of neurogenic lesion, while those with SBA are often diagnosed with severe degree of neurogenic lesion. These findings stress the fact that for those patients with SBO, clinical presentation is usually with mild manifestations and therefore there is a great need for timely diagnosis of this condition in order to prevent possible complications. Frequent findings of severe degree of neurogenic lesion in patients with SBA stress the necessity for timely diagnosis and a multidisciplinary approach in the treatment plan for these patients.

In conclusion, patients with SBO usually have a mild to moderate clinical presentation, while multiple root involvement and severe degree of neurogenic lesion are associated more frequently with SBA.

\section{References}

1. Padmanabhan R. Etiology, pathogenesis and prevention of neural tube defects. Congenit Anom (Kyoto) 2006; 46: 55-67.

2. Tortori-Donati P, Rossi A, Cama A. Spinal dysraphism: a review of neuroradiological features with embryological correlations and proposal for a new classification. Neuroradiology 2000; 42: 471-91.

3. Tsai PY, Cha RC, Yang TF, et al. Electromyographic evaluation in children with spina bifida. Zhonghua Yi Xue Za Zhi (Taipei) 2001; 64: 509-15.

4. de Wals P, Tairou F, van Allen MI, et al. Reduction in neural-tube defects after folic acid fortification in Canda. N Engl J Med 2007; 357: 135-42.

5. Nikolic D, Cvjeticanin S, Petronic I, et al. Degree of genetic homozygosity and distribution of $A B O$ blood types among patients with spina bifida occulta and spina bifida apperta. Arch Med Sci 2010; 6: 854-9.

6. Stalberg E. Advanced needle EMG methods. In: Johnson's practical electromyography. Pease WS, Lew HL, Johnson EW (eds). 4th ed. Philadelphia (Pennsylvania): Lippincott Williams and Wilkins 2007; 105-30.

7. Curt A, Dietz V. Electrophysiological recordings in patients with spinal cord injury: significance for predicting outcome. Spinal Cord 1999; 37: 157-65.

8. Kimura J. Types of abnormality. In: Electrodiagnosis in diseases of nerve and muscle: principles and practice. Kimura J (ed.). Philadelphia, PA FA Davis Company 1981; 258-9.

9. Preston DC, Shapiro BE. Needle electromyography. Fundamentals, normal and abnormal patterns. Neurol Clin 2002; 20: 361-96.

10. Dias MS. Neurosurgical management of myelomeningocele (spina bifida). Pediatr Rev 2005; 26: 50-60.

11. Guggisberg D, Hadj-Rabia S, Viney C, et al. Skin markers of occult spinal dysraphism in children: a review of 54 cases. Arch Dermatol 2004; 140: 1109-15.

12. Krogh K, Christensen P. Neurogenic colorectal and pelvic floor dysfunction. Best Pract Res Clin Gastroenterol 2009; 23: 531-43.

13. Hong CZ. Electromyographic assessment of neurological function in patients with myelomeningocele caused by spina bifida. Zhonghua Yi Xue Za Zhi (Taipei) 2001; 64: 516-8. 
14. Lemelle JL, Guillmenin F, Aubert D, et al. A multicentre study of the management of disorders of defecation in patients with spina bifida. Neurogastroenterol Motil 2006; 18: 123-8.

15. Vande Velde S, van Biervliet S, van Renterghem K, et al. Achieving fecal continence in patients with spina bifida: a descriptive cohort study. J Urol 2007; 178: 2640-4.

16. Yuan Z, Cheng W, Hou A, et al. Constipation is associated with spina bifida occulta in children. Clin Gastroenterol Hepatol 2008; 6: 1348-53. 